\title{
REMOÇÃO DO CORANTE REATIVO REMAZOL VERMELHO EMPREGANDO O PROCESSO DE ADSORÇÃO
}

\author{
L. C. PAVAN ${ }^{1}$, R. L. S. CANEVESI ${ }^{1}$, D. MOLINARI ${ }^{1}$, C. E. BORBA ${ }^{1}$, M. G. A. VIEIRA ${ }^{2}$, \\ E. A. da SILVA ${ }^{2}$ \\ ${ }^{1}$ Universidade Estadual do Oeste do Paraná, Centro de Engenharia e Ciências Exatas \\ 2 Universidade Estadual de Campinas, Faculdade de Engenharia Química \\ E-mail para contato: linpavan@hotmail.com
}

\begin{abstract}
RESUMO - Com o crescimento industrial alcançado nos últimos anos, ocorreu um aumento significativo na quantidade de rejeitos industriais, dentre eles, destaca-se os efluentes têxteis com características tóxicas, cancerígenas e mutagênicas. Desta maneira, o objetivo principal deste trabalho foi investigar a adsorção em batelada do corante reativo Remazol Vermelho. Foram realizados ensaios em batelada para avaliar a capacidade de adsorção dos seguintes adsorventes: argila organofilizada comercial, argila esmectita natural, escamas de peixe, carvão vegetal e de ossos e seguido da avaliação da influencia dos efeitos do $\mathrm{pH}$ inicial da solução na faixa de 1 a 6 e temperatura na faixa de 15 a $45^{\circ} \mathrm{C}$. O carvão de osso foi o adsorvente que apresentou maior capacidade de remoção do corante. Os resultados da análise estatística mostraram que o $\mathrm{pH}$ inicial da solução apresentou influencia significativa na capacidade de adsorção. $\mathrm{O}$ pH inicial igual a 1 apresentou maior remoção de corante dentro da faixa investigada.
\end{abstract}

\section{INTRODUÇÃO}

O setor têxtil tem apresentado uma elevada taxa de desenvolvimento, principalmente com o aumento na utilização de corantes sintéticos pela sociedade moderna (SOLIS et al., 2012). Contudo, o efluente líquido oriundo deste setor se tornou um dos principais potenciais contaminantes de recursos naturais, como rios e lagos, alcançando a marca de 150 milhões de metros cúbicos de efluente produzidos mundialmente por ano (IP et al., 2010).

Os corantes, mesmo em baixas concentrações apresentam características tóxicas, carcinogênicas, mutagênicas e teratogênicas, dessa forma, representando um grave risco à saúde humana (KUMAR et al., 2010), além de afetar negativamente a fauna e a flora aquática (MEZOHEGYI \& ZEE, 2012).

O processo de adsorção vem se destacando em virtude das altas capacidades de remoção de corantes de meios aquosos, simplicidade de projeto, implantação e operação (PANIC \& VELICKOVIC, 2014).

Desta forma, o presente estudo tem como objetivo: avaliar a eficiência dos adsorventes Argila organofilizada comercial, argila esmectita natural, Carvão Vegetal, carvão de ossos e escamas de peixe, na remoção do corante reativo Remazol Vermelho (RV), bem como 


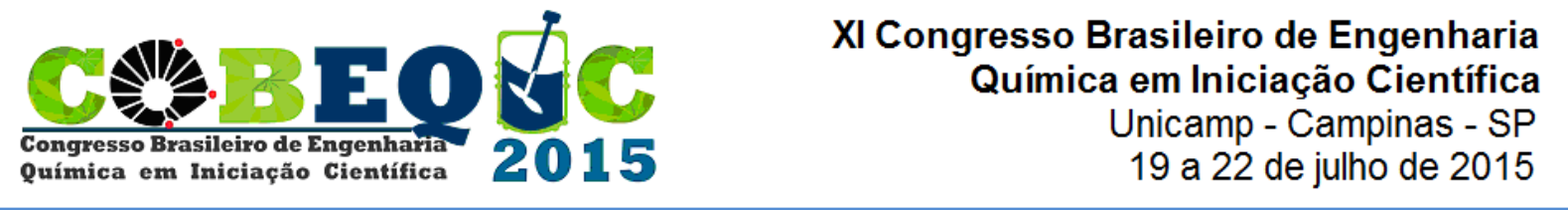

identificar a influencia dos fatores $\mathrm{pH}$ inicial da solução e temperatura de operação na capacidade de adsorção.

\section{MATERIAIS E METODOS}

\subsection{Adsorventes}

Os adsorventes utilizados neste trabalho foram: Argila organofilizada comercial Spectrogel adquirida da empresa Spectrochem ${ }^{\circledR}$, argila esmectita natural proveniente de Boa Vista - Paraíba, Carvão Vegetal comercial fornecido pela empresa Alphacarbo, carvão de ossos fornecido pela empresa Bonechar e escamas de peixe da espécie Oreochomis niloticus (Tilápia do Nilo), cedidas pela Cooperativa COPISCES.

\subsection{Solução de Corante}

As soluções de corante empregadas foram preparadas a partir da dissolução do corante em pó Remazol Vermelho (RV) da marca Dystar em água destilada. O pH das soluções foi ajustado com a adição de ácido clorídrico (P.A.) e hidróxido de sódio (P.A.).

\subsection{Seleção do Adsorvente}

Os testes foram realizados em batelada sob agitação de $100 \mathrm{rpm}$ em uma mesa agitadora marca Tecnal, na temperatura de $30^{\circ} \mathrm{C}$ e $\mathrm{pH}$ inicial igual a 2. Para que o efeito da granulometria não apresente influencia nos ensaios de adsorção, empregou-se os adsorventes na granulometria menor que 0,1 mesh. Em erlenmeyers de $125 \mathrm{~mL}$ adicionou-se $75 \mathrm{~mL}$ de solução de corante na concentração de $150 \mathrm{mg} \mathrm{L}^{-1}$ e $0,1 \mathrm{~g}$ de adsorvente, empregando tempo de contato de 48 horas. Os Adsorventes foram separados da fase liquida por centrifugação durante $10 \mathrm{~min}$. Os testes foram realizados em quintuplicatas.

A concentração na fase sólida dos corantes foram calculadas a partir do balanço de massa representado pela seguinte equação.

$$
q=\frac{V\left(C_{0}-C_{f}\right)}{m}
$$

Em que: $q$ consiste na concentração de corante na fase sólida em $\mathrm{mg} \mathrm{g}^{-1}, C_{0}$ e $C_{f}$ representam as concentrações de corante na fase fluída, inicial e final respectivamente em $\mathrm{mg} \mathrm{L} \mathrm{L}^{-1}, m$ a massa de adsorvente usada em g e $V$ o volume de solução em L.

As concentrações iniciais e remanescentes foram determinadas empregando espectrofotometria UV/Vis no comprimento de onde de $514 \mathrm{~nm}$ determinado à partir de levantamento do espectro de absorbância na faixa de 900 a $190 \mathrm{~nm}$. Para a escolha do adsorvente de melhor desempenho na remoção dos corantes foi empregado a metodologia estatística de teste Fischer LSD.

\subsection{Efeito do pH e da Temperatura}


Para avaliar a influência dos fatores temperatura e $\mathrm{pH}$ inicial da solução na quantidade de corante adsorvido, empregando o adsorvente que apresentou melhor desempenho nos testes preliminares realizou-se um planejamento experimental do tipo delineamento composto central rotacional (DCCR) com 5 replicatas no ponto central. Os níveis avaliados são apresentados na Tabela 1 .

Tabela 1. Níveis Investigados no planejamento experimental DCCR

\begin{tabular}{ccc}
\hline Níveis & Temperatura & $\mathrm{pH}$ \\
\hline$(-1,41)$ & 16 & 1 \\
$(-1,0)$ & 20 & 2 \\
$(0,0)$ & 30 & 3,5 \\
$(1,0)$ & 40 & 5 \\
$(1,41)$ & 45 & 6 \\
\hline
\end{tabular}

Os testes foram realizados numa mesa agitadora orbital com controle de temperatura marca Tecnal sobre agitação constante de $100 \mathrm{rpm}$, empregando um tempo de contato de 48 horas, volume de solução no reator de $75 \mathrm{~mL}$, na concentração de $150 \mathrm{mg} \mathrm{L}^{-1}$ e 0,1 gramas de adsorvente. $\mathrm{O}$ Adsorvente foi separado da fase liquida a partir de centrifugação durante 10 min. As concentrações iniciais e remanescentes foram determinadas empregando espectrofotometria UV/Vis no comprimento de onde de $514 \mathrm{~nm}$.

\section{RESULTADOS E DISCUSSÕES}

\subsection{Seleção do Adsorvente}

Foram realizados experimentos em sistema batelada, com agitação de 100 rpm, e pH da solução em 2, utilizando o corante RV, com o intuito de avaliar qual adsorvente entre os testados foi mais eficiente na remoção do corante. Na Figura 1 são apresentados os resultados da concentração corante Remazol Vermelho no adsorvente após o tempo de contato de 48 horas.

O teste estatístico de Fisher LSD de comparação de média, foi utilizado para identificar o adsorvente que apresentou maior capacidade de remoção. Para o nível de significância de $5 \%$ o carvão de ossos foi o adsorvente que apresentou maior capacidade de adsorção, seguido da escama de peixe. Portanto, o adsorvente que apresentou maior eficiência na remoção do corante RV foi o carvão de Ossos. 


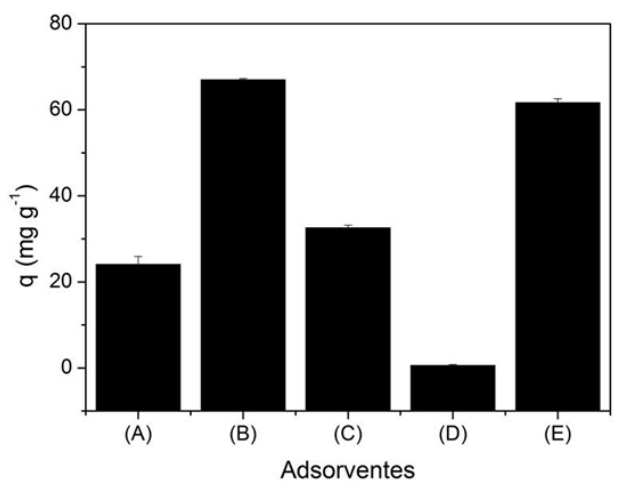

Figura 1. Concentração no adsorvente no equilíbrio para diferentes adsorventes: (A)

Carvão Vegetal; (B) Carvão de Ossos; (C) Argila Organofílica Spectrogel; (D) Argila Esmectita Natural; (E) Escama de Peixe.

\subsection{Efeito do pH e da Temperatura}

Com o intuito de avaliar o efeito do $\mathrm{pH}$ e da temperatura no processo de adsorção, empregando como adsorvente o carvão de ossos, aplicou-se a metodologia de planejamento experimental DCCR, com uma quintuplicata no ponto central. O comportamento da capacidade de remoção do corante Remazol vermelho em função da temperatura e pH é apresentado na Tabela 3 .

Tabela 3. Capacidades de remoção em função do pH e da Temperatura no processo de adsorção do corante Remazol Vermelho.

\begin{tabular}{ccc}
\hline Temperatura & $\mathbf{p H}$ & $\mathbf{q ( \mathbf { m g ~ g } ^ { \mathbf { - 1 } } )}$ \\
\hline 40 & 2 & 86,76 \\
20 & 2 & 75,74 \\
40 & 5 & 38,42 \\
20 & 5 & 28,22 \\
16 & 3,5 & 26,00 \\
44 & 3,5 & 39,35 \\
30 & 1 & 81,22 \\
30 & 6 & 35,20 \\
30 & 3,5 & 35,95 \\
30 & 3,5 & 36,42 \\
30 & 3,5 & 36,38 \\
30 & 3,5 & 36,02 \\
30 & 3,5 & 36,49 \\
\hline
\end{tabular}

Os efeitos obtidos para cada fator na adsorção do corante Remazol Vermelho é apresentado na Tabela . 
Tabela 4. Efeitos obtidos no processo de adsorção do corante Remazol Vermelho.

\begin{tabular}{ccccc}
\hline & Efeito & $\begin{array}{c}\text { Erro } \\
\text { Padrão }\end{array}$ & p-valor & Coeficiente \\
\hline Media & 37,19 & 4,58 & $8,34 \mathrm{E}-05$ & 37,19 \\
Temperatura(L) & 10,02 & 7,28 & $2,11 \mathrm{E}-01$ & 5,01 \\
Temperatura(Q) & 3,89 & 7,90 & $6,38 \mathrm{E}-01$ & 1,94 \\
pH(L) & $-36,12$ & 6,66 & $9,86 \mathrm{E}-04$ & $-18,06$ \\
pH(Q) & 19,49 & 5,97 & $1,38 \mathrm{E}-02$ & 9,75 \\
1L by 2L & $-0,41$ & 10,30 & $9,69 \mathrm{E}-01$ & $-0,21$ \\
\hline
\end{tabular}

De acordo com a Tabela 4, a variação do fator temperatura não foi significativa na capacidade de adsorção do corante Remazol Vermelho nas suas duas formas, linear (L) e quadrática (Q), para o nível de significância estudado. O modelo estatístico obtido e representado matematicamente pela equação (2):

$$
q=38,6-18,0 p H+9,4 p H^{2}
$$

Para avaliar a eficiência do modelo estatístico empregado na analise dos dados experimentais de adsorção, utiliza-se a metodologia de analise de variância, apresentada na Tabela .

Comparando o valor de $\mathrm{F}$ tabelado para os graus de liberdade, no valor de 4,1, com o valor apresentado na Tabela, nota-se que o valor obtido é 5 vezes maior que o valor tabelado, o que indica que o modelo estatístico empregado conseguiu descrever apropriadamente os efeitos destas variáveis na adsorção do corante Remazol Vermelho.

Tabela 5. Analise de variância (ANOVA) aplicada à metodologia DCCR do processo de adsorção do corante Remazol Vermelho.

\begin{tabular}{cccccc}
\hline & SQ & GL & MQ & F & $\mathrm{R}^{2}$ \\
\hline Modelo & 4224,6 & 2 & 2112,3 & 21,8 & 0,81 \\
Erro & 969,0 & 10 & 96,9 & & \\
Total & 5193,6 & 12 & & & \\
\hline
\end{tabular}

Thangamani et al., (2007) e Mao et al. (2009), que obtiveram em seus estudos sobre o efeito do $\mathrm{pH}$ na remoção de corantes aniônicos (reativos) comportamento semelhante ao obtido no presente estudo. Já para Aravindhan et al. (2007) corantes aniônicos competem com os íons ${ }^{-} \mathrm{OH}$, da solução pelos sítios ativos da superfície do adsorvente, não acontecendo este tipo de competição em meios ácidos, o que favorece a adsorção do corante.

\section{CONCLUSÃO}

Neste trabalho investigou-se o processo de adsorção do corante Remazol Vermelho. Constatou-se inicialmente que dos adsorventes testados a maior capacidade de adsorção do corante Remazol Vermelho foi obtida empregando o Carvão de Ossos. A análise dos resultados do planejamento experimental DCCR mostrou que na faixa de $\mathrm{pH}$ estudada, 
adsorção é favorecida pelo decréscimo do $\mathrm{pH}$, sendo que a maior capacidade adsortiva obtida foi no $\mathrm{pH}$ igual a 1, A metodologia DCCR também mostrou que para o nível de significância estudada $(\alpha=5 \%)$ a temperatura do processo não se mostrou significativa para o corante RV. A alta capacidade de adsorção torna o carvão de ossos uma boa alternativa para o tratamento de efluentes que contenham este tipo de corante.

\section{REFERENCIAS BIBLIOGRAFICAS}

ARAVINDHAN, R.; RAO, J. R.; NAIR, B. U.. Removal of basic yellow dye from aqueous solution by sorption on Green alga Caulerpa Scalpelliformis. J. Hazard Maters, 142, p. 68-76, 2007.

IP, A. W. M., BARFORD, J. P., MCKAY, G.. A comparative study on the kinetics and mechanisms of removal of Reactive Black 5 by adsorption onto activated carbons and bone char. Chemical Engineering Journal,157, p. 434-442, 2010.

KUMAR, P. S., RAMALINGAM S., SENTHAMARAI, C., NIRANJANAA, M., VIJAYALAKSHMI, P., SIVANESAN, S.. Adsorption of dye from aqueous solution by cashew nut shell: studies on equilibrium isotherm, kinetics and thermodynamics of interactions. Desalination, 261, p. 52-60, 2010.

MAO, J.; WON, S. W.; VIJAYARAGHAVAN, K. YUN, Y.. Surface modification of Corynebacterium glutamicum for enhanced Reactive Red 4 biosorption. Biosource Technology, 100, p. 1463-1466, 2009.

MEZOHEGYI, G., van der ZEE, F.P., FONT, J., FORTUNY, A., FABREGAT, A.. Towards advanced aqueous dye removal processes: a short review on the versatile role of activated carbon. J. Environ. Manage., 102, p. 148-164, 2012.

PANIC, V. V., VELICKOVIC, S. J.. Removal of model cationic dye by adsorption onto poly(methacrylic acid)/zeolite hydrogel composites: Kinetics, equilibrium study and image analysis. Separation and Purification Technology, 122, p. 384-394, 2014.

SOLÍS, M., SOLÍS, A., PÉREZ, H.I., MANJARREZ, N., FLORES, M.. Microbial decolouration of azo dyes: a review. Process Biochem. 47, p. 1723-1748, 2012.

THANGAMANI, K. S., SATHISHKUMAR, M., SAMEENA, Y., VENNILAMANI, N., KADIRVELU, K., PATTABHI, S., YUN, S. E., Utilization of modified silk cotton hull waste as an adsorbent for the removal of textile dye (reactive blue MR) from aqueous solution, Bioresource Technology, 98, 1265-1269, 2007. 